\title{
Unique Elution Behavior of Bromide and Nitrate in Anion-Exchange Chromatography Using Aqueous Potassium Chloride Eluent Containing Cadmium or Zinc Ion
}

\author{
Masahiro MARUO ${ }^{* *}$ and Hajime OBATA** \\ *School of Environmental Science, The University of Shiga Prefecture, Hassaka, Hikone, \\ Shiga 522-8533, Japan \\ **Atmosphere and Ocean Research Institute, The University of Tokyo, Kashiwanoha, Kashiwa, \\ Chiba 277-8564, Japan
}

\begin{abstract}
In anion-exchange chromatography using a high-concentration eluent and high-capacity ion-exchange resin, the effect of the countercation contained in the eluent was investigated. Cadmium(II) and zinc(II) ions were examined as additives in an aqueous potassium chloride eluent. The addition of these cations resulted in a reversed elution order of bromide and nitrate, as compared with conventional anion-exchange chromatography. The separation factor for these two anions increased as the cadmium concentration in the eluent was increased. Zinc(II) ion was also effective, but a relatively high concentration was necessary.
\end{abstract}

(Received March 31, 2011; Accepted July 25, 2011; Published September 10, 2011)

\section{Introduction}

In anion-exchange chromatography, the effect of the countercation in the eluent ${ }^{1,2}$ has not been thoroughly investigated because the eluent concentration is typically on the order of millimoles per liter, and the countercation is considered to have little effect on the retention of analyte anions. To change the elution order of anions, the water molecules that solvate the analyte anions should be removed to enhance the interaction between the analytes and the ion-exchange groups. For this purpose, the stationary phase can be changed to alumina or ion-exchange resin with amino groups that form ammonium $\left(-\mathrm{NH}_{3}{ }^{+}\right){ }^{3} \quad$ Furthermore, when using a conventional anion-exchange group, such as $-\mathrm{NEt}_{3}{ }^{+}$, a considerable amount of aprotic organic solvent, such as acetonitrile, is necessary in the eluent. Through such approaches, the elution order of halogen anions can be reversed. ${ }^{4}$ Hirayama et al. investigated immobilized charged complex anion-exchange groups containing transition-metal cations, such as copper(II) and cadmium(II). This approach was successful in changing the elution order of inorganic anions, but the eluent anion was limited to benzenesulfonate to minimize its interaction with metal cations retained on ion-exchange groups. ${ }^{5-7}$

Our laboratory has reported that the separation factor between bromide and nitrate can be modified through the use of alkaline metal cations along with a high-concentration eluent and high-capacity anion-exchange resin. ${ }^{8}$ This method was used to determine nitrate in seawater. When using alkaline and alkaline earth metal cations in the previous study, the separation factor of nitrate relative to bromide was found to depend on both the

† To whom correspondence should be addressed.

E-mail:maruo@ses.usp.ac.jp countercation used and its concentration. However, the elution order of these anions was not changed.

In the present study, we further investigated the effect of other countercations in the eluent in order to enhance the selectivity for bromide and nitrate in anion-exchange chromatography using a conventional resin as the stationary phase. In these experiments, unique effects of cadmium and zinc ions in the eluent were observed.

\section{Experimental}

\section{Reagents}

Standard stock solutions were prepared by dissolving analytical-grade sodium nitrite, sodium nitrate, and sodium bromide (Wako Chemicals, Japan) in ultrapure water (Mill-Q). Working standard solutions were prepared by diluting each stock solution with ultrapure water. Seawater samples were collected from the North Eastern Pacific Ocean.

The eluent was prepared by dissolving analytical-grade potassium chloride, zinc chloride, and cadmium chloride (Wako) in ultrapure water. The eluent was degassed by ultrasonication before use. After dissolution of the reagents, the $\mathrm{pH}$ of the eluent was adjusted in the range from 3.6 to 9.3 by adding a small amount of aqueous hydrochloric acid or aqueous ammonia.

\section{Apparatus}

The ion-exchange chromatography system consisted of a Shimadzu LC10-ADvp double plunger pump (flow rate, $0.25 \mathrm{~mL} \mathrm{~min}^{-1}$ ), a Rheodyne 9125 PEEK injection valve (injection volume, $20 \mu \mathrm{L}$ ), a Shimadzu SPD-10AVi UV-visible detector, and a Yokogawa 3056 pen recorder. The detection wavelength was fixed at $220 \mathrm{~nm}$. The column was kept at ambient room temperature (approximately $25^{\circ} \mathrm{C}$ ). The separation 
(a)

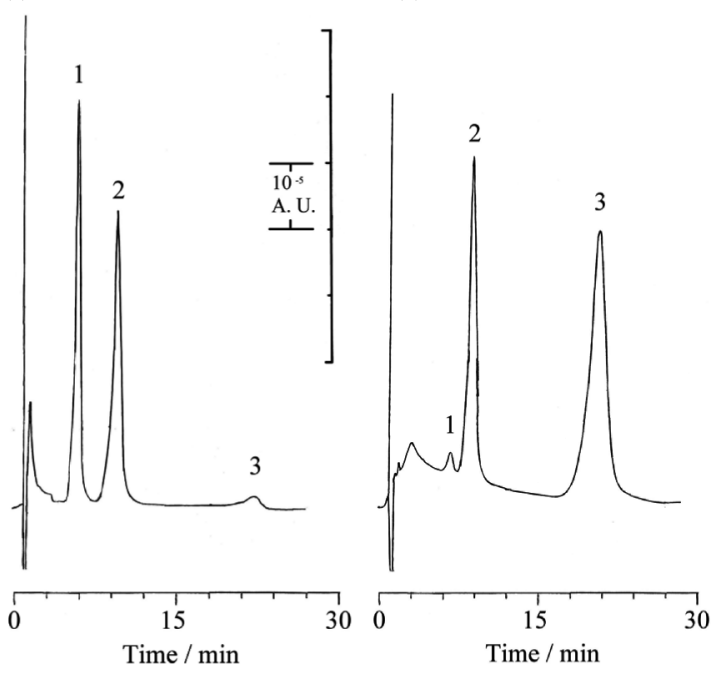

Fig. 1 Typical chromatograms of nitrite, bromide, and nitrate when using aqueous potassium chloride eluent containing cadmium chloride. (a) Eluent: $1 \mathrm{~mol} \mathrm{~L}^{-1} \mathrm{KCl}$ and $1 \mathrm{mmol} \mathrm{L}^{-1} \mathrm{CdCl}_{2}$ at $\mathrm{pH} 9.3$. Sample: mixed standard solution of nitrite, nitrate and bromide in MWQ. The concentration of each anions is $20 \mu \mathrm{mol} \mathrm{L}^{-1}$. (b) Eluent: the same composition for (a) at pH 3.6. Sample: seawater at the North Eastern Pacific Ocean (depth: $175 \mathrm{~m}$ ). Anions: 1, nitrite; 2, nitrate; 3, bromide. The nitrate concentration was $25.3 \mu \mathrm{mol} / \mathrm{L}$, determined by a continuous flow analyzer and spectrophotometry onboard.

column was prepared by packing Tosoh TSKgel SAX (ion-exchange capacity, $1.0 \mathrm{meq} \mathrm{g}^{-1}$; diameter, $5 \mu \mathrm{m}$ ) into a PEEK-lined stainless-steel column $(2.0 \mathrm{~mm}$ i.d. $\times 100 \mathrm{~mm}$, Upchurch, USA) with a maximum operating pressure of $10 \mathrm{MPa}$.

\section{Results and Discussion}

Effect of cadmium ion in potassium chloride eluent on the retention of analyte anions

As previously reported, the separation factor of nitrate relative to bromide in $1 \mathrm{~mol} \mathrm{~L}^{-1}$ aqueous potassium chloride eluent was found to be 1.18 , the smallest value obtained among the alkaline and alkaline earth metal chlorides tested as eluent at the same chloride concentration ( $\mathrm{LiCl}$ gave the highest separation factor: 1.55). ${ }^{8}$ By using alkaline and alkaline earth metal chlorides, the selectivity for each analyte was enhanced by decreasing the concentration of the eluent anion, but the order of elution could not be changed.

However, upon adding of cadmium chloride to the aqueous potassium chloride eluent, the elution order for these anions was reversed without the addition of an organic solvent, and bromide was eluted after nitrate. Typical chromatograms are shown in Fig. 1. The dependence of the adjusted retention time $\left(t_{\mathrm{R}}{ }^{\prime}\right)$ of the analytes on the cadmium ion concentration in the eluent is given in Fig. 2. The separation factor of bromide relative to nitrate (i.e., the inverse value of the separation factor of nitrate relative to bromide) increased from 0.87 to 2.64 with the addition of $1 \mathrm{mmol} \mathrm{L}^{-1}$ cadmium chloride into $1 \mathrm{~mol} \mathrm{~L}^{-1}$ potassium chloride eluent at $\mathrm{pH}$ 3.6. With an increase in the $\mathrm{Cd}^{2+}$ concentration, $t_{\mathrm{R}}{ }^{\prime}$ of $\mathrm{NO}_{3}{ }^{-}$was decreased, and that of $\mathrm{Br}$ was increased, while $t_{\mathrm{R}}{ }^{\prime}$ of $\mathrm{NO}_{2}{ }^{-}$did not show any significant change.

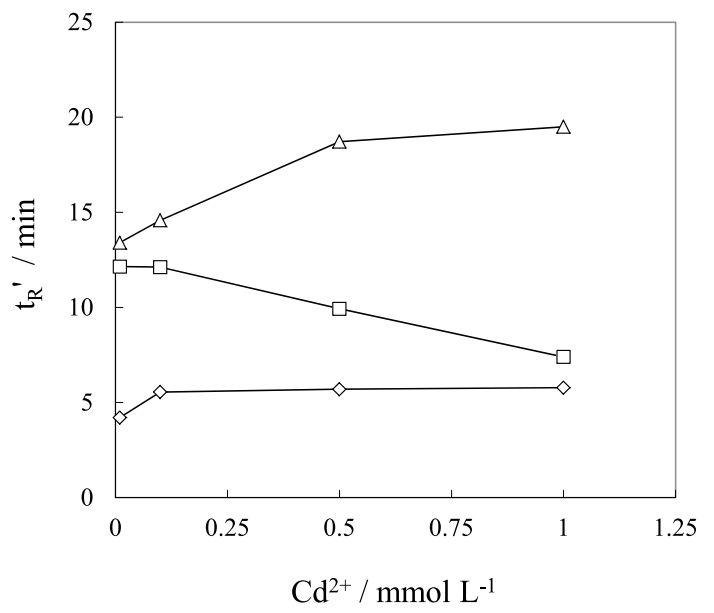

Fig. 2 Adjusted retention times of analytes versus cadmium concentration in aqueous potassium chloride eluent $\left(1 \mathrm{~mol} \mathrm{~L}^{-1}\right)$ at $\mathrm{pH}$ 3.6: nitrite (diamond), nitrate (square), and bromide (triangle).

Aluminum and lanthanum chlorides $\left(1 \mathrm{~mol} \mathrm{~L}^{-1}\right.$ chloride $)$ were also tested, and showed higher separation factors $\left(\mathrm{AlCl}_{3}, 1.48\right.$; $\left.\mathrm{LaCl}_{3}, 1.38\right)$, but the order of elution was not changed.

\section{Complex formation of cadmium ion with anions}

The increase in the retention time of bromide with increasing cadmium concentration suggests that bromide interacts with cadmium. In the literature, the stability constants of cadmium ion complexation with bromide in $0.75 \mathrm{~mol} \mathrm{~L}^{-1}$ sodium perchlorate at $25^{\circ} \mathrm{C}$ are as follows: $\log K_{1}, 1.53$; $\log \beta_{2}, 2.00$; $\log \beta_{3}, 2.29 ; \log \beta_{4}, 2.45$. $^{9}$ In the case of bromide, the formation of an anionic complex with cadmium ion might retard the elution of bromide to some extent, although chloride in the eluent also undergoes complexation with cadmium ion. The stability constant $\left(\log K_{1}\right)$ of cadmium complexation with nitrate in $2 \mathrm{~mol} \mathrm{~L}^{-1}$ sodium perchlorate at $25^{\circ} \mathrm{C}$ is small $\left(\log K_{1}=0.11\right),{ }^{9}$ and the successive stepwise stability constants are much smaller. This suggests that the cadmium ion partly interacts with nitrate, reducing its effective anionic charge; the retention time of nitrate decreased as the cadmium ion concentration in the eluent was increased.

\section{Effect of the eluent $\mathrm{pH}$ on the retention of anions}

The effects of the eluent $\mathrm{pH}$ were investigated at 3.6, 4.8, 6.5, and 9.3 , by adding small amounts of aqueous hydrochloric acid or aqueous ammonia to the eluent solution. As can be seen in chromatograms in Figs. 1(a) and 1(b), no significant change was observed in the retention times and the separation factors of the analytes, except for nitrite at $\mathrm{pH} 3.6$, which was probably caused by a decrease in the net charge $\left(\mathrm{p} K_{\mathrm{a}}=3.14\right){ }^{10}$ These results show that there was almost no change in the ionic form of these analytes in the $\mathrm{pH}$ range tested.

Effect of potassium chloride concentration on the retention of anions

To investigate the effects of the eluent concentration on the retention time of analytes, the eluent concentration (for only potassium chloride) was varied in the range from 0.5 to $2 \mathrm{~mol} \mathrm{~L}^{-1}$. The slope of plots of $\log t_{\mathrm{R}}{ }^{\prime}$ versus $-\log$ [eluent] approximately represents the ratio of the charge of the analyte anion to that of the eluent anion (chloride). ${ }^{1}$ In this experiment, the effect of additional chloride from the addition of cadmium 


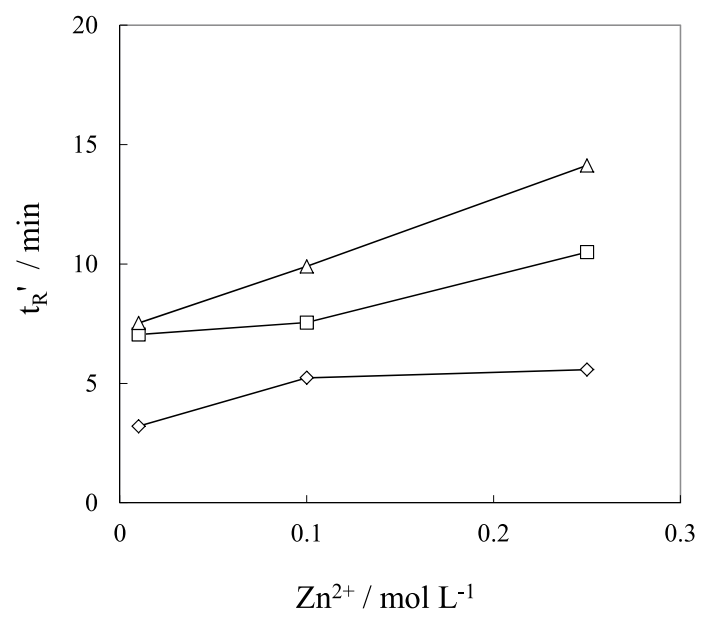

Fig. 3 Adjusted retention times of analytes versus the zinc ion concentration in aqueous potassium chloride eluent (total chloride: $1 \mathrm{~mol} \mathrm{~L}^{-1}$ ) at $\mathrm{pH}$ 3.6: nitrite (diamond), nitrate (square), and bromide (triangle)

chloride was ignored. Theoretically, the slopes for all anions in this study were expected to be -1 . The obtained slopes were -1.08 for bromide and -1.10 for nitrate at $\mathrm{pH} 3.6$ when potassium chloride was used as the eluent with $1 \mathrm{mmol} \mathrm{L}^{-1}$ cadmium chloride. For nitrite, a straight line was not obtained, probably due to the protonation of nitrite at low $\mathrm{pH}$. At $\mathrm{pH} 9.3$, the obtained slopes were $-0.83,-1.10$, and -0.99 for bromide, nitrate, and nitrite, respectively. In our previous study, when aqueous lithium chloride was used as the eluent within the same concentration range, the slopes were $-0.85,-0.88$, and -0.85 , and when aqueous sodium chloride was used as the eluent, the slopes were $-0.83,1.04$, and -0.74 , respectively. Because the slope for nitrate in sodium chloride eluent was higher than that in lithium chloride eluent, the nitrate ion was affected by ion-pair formation with countercations, including potassium ion. In the experiment using cadmium as an additive in potassium chloride eluent, the retention time of nitrate was found to decrease as a result of complexation or ion-pair formation with potassium and cadmium ion, which decreased the effective charge of nitrate. As shown in Fig. 2, only cadmium ion was effective in decreasing $t_{\mathrm{R}}{ }^{\prime}$ of nitrate, but the net charge of nitrate was considered to be affected by the extent of ion-pair formation with increasing potassium ion concentration.

Bromide appeared to be less strongly affected by ion-pair formation with countercations. As shown by the increase in $t_{\mathrm{R}}{ }^{\prime}$ with additional cadmium ion, multiple bromide ions might be involved in complexation with the cadmium ion.

Effect of zinc ion in eluent on the retention of analyte anions Similar elution behavior for bromide and nitrate in cadmium-containing eluent was also observed when zinc ion was added to the eluent. In this experiment, the $\mathrm{pH}$ was fixed at 3.6 to avoid precipitation of zinc hydroxide. The chloride concentration was held constant at $1 \mathrm{~mol} \mathrm{~L}^{-1}$, while the concentrations of potassium ion and zinc ion were varied. As shown in Fig. 3, the order of elution was the same as when cadmium ion was added to the eluent, and the separation factor of bromide relative to nitrate increased as the concentration of zinc ion in the eluent was increased. However, a high concentration of zinc ion was required in the eluent, and a reduction in the retention time of nitrate was not observed, in contrast to the case of cadmium-containing eluent. Metallic cadmium and zinc are used to reduce nitrate to nitrite for the spectrophotometric determination of nitrate in aqueous solutions; ;,11,12 therefore, this elution behavior might provide insight into the reduction mechanism of nitrate on these metals.

Hirayama et al. ${ }^{7}$ investigated the selectivity of anions $\left(\log k^{\prime} / k^{\prime}\right)$, and found unique selectivity when anion-exchange groups with immobilized copper(II) and cadmium(II) were used. In the literature, nitrate is reported to elute faster than bromide, as observed in the present experiment using these two metal ions as additives. With the use of zinc(II), the selectivity of these anions was normal and seemed to be rather poor, but we found that the selectivity could be changed by adjusting the concentration of metal ion, as shown in Fig. 3.

\section{Conclusions}

The addition of cadmium(II) ion into aqueous potassium chloride eluent in anion-exchange chromatography resulted in a reversed elution order of bromide and nitrate. The separation factor for these two anions increased as the cadmium concentration in the eluent was increased. Zinc(II) ion was also effective in reversing the elution order of these anions, but a relatively high concentration was necessary. An advantage of using these metal ions as additives in the eluent is that their concentration and mixing ratio with other metals can be easily adjusted, compared with use in the stationary phase.

Since, cadmium and zinc cations have low absorption in the UV range, these phenomena will be applicable in the determination of these anions in samples with high concentrations of other anions (e.g., determining nitrate in seawater; Fig. 1(b)). Studies on the application of this method are now in progress.

\section{References}

1. P. R. Haddad and P. E. Jackson, "Ion Chromatography", 1990, Elsevier, Amsterdam.

2. American Public Health Association, "Standard Methods for the Examination of Water and Wastewater", 19th ed., 1995, John Wiley \& Sons, Inc., New York, Part 4000, $4-85$.

3. T. Okada, Bunseki Kagaku, 1995, 44, 579.

4. T. Okada, J. Chromatogr., A, 1997, 758, 19.

5. N. Hirayama and W. Umehara, Anal. Chim. Acta, 1996, $334,1$.

6. N. Hirayama, Bunseki Kagaku, 1999, 48, 33.

7. N. Hirayama, W. Umehara, H. Makizawa, and T. Honjo, Anal. Chim. Acta, 2000, 409, 17.

8. M. Maruo, T. Doi, and H. Obata, Anal. Sci., 2006, 22, 1175.

9. E. Hogfeldt Compiled, "Stability Constants of Metal-Ion Complexes, IUPAC Chemical Data Series No. 21, Part A: Inorganic Ligands", 1982, Pergamon Press, Oxford, New York, Toronto, Sydney, Paris, Frankfurt, 252.

10. J. A. Dean, "Lange's Handbook of Chemistry", 15th ed., 1999, McGraw-Hill, New York.

11. E. Morita and E. Nakamura, Bunseki Kagaku, 2008, 57, 777.

12. Japan Industrial Standard K1012 43.2.3, 1998. 